\title{
A WILD SPHERE WHICH CAN BE PIERCED AT EACH POINT BY A STRAIGHT LINE SEGMENT
}

\author{
M. K. FORT, JR. ${ }^{1}$
}

In this note we describe a wild 2 -sphere $S$ in $E^{3}$ which can be pierced at each point by a straight line segment. The existence of such a wild, sphere answers the following question which was raised by R. H. Bing in his address Embedding surfaces in 3-manifolds at the 1962 International Congress of Mathematicians in Stockholm:

Is a 2-sphere in $E^{3}$ tame if it can be pierced at each point by a straight line interval?

In order to make the description of $S$ as concise as possible, we make use of the terminology and notation of [2], the author's modification of Bing's well-known "Dog Bone Decomposition" (see [1]). We let $G$ be the decomposition space defined in [2], and let $A_{0}$ be the union of the nondegenerate elements of $G$. It is possible to carry out the construction of $A_{0}$ in such a way that the endpoints of the components of $A_{0}$ lie in two parallel planes, and we assume that this is the case. We can represent $A_{0}=\bigcap_{n=0}^{\infty} B_{n}$, where each set $B_{n}$ is the union of $2^{n}$ admissible polyhedra (see [2, p. 502, Figure 1]) $P_{1}^{n}, P_{2}^{n}$, $\cdots, P_{2^{n}}^{n}$. Now, if (in the notation of [2]) $P_{j}^{n}$ is represented as $L \cup M \cup R$, we define $Q_{j}^{n}$ to be (basic parallelepiped of $\left.L\right) \cup M \cup$ (basic parallelepiped of $R$ ). We now define $S$ to be the boundary of

$$
\bigcup_{n=0}^{\infty} \bigcup_{j=1}^{2^{n}} Q_{j}^{n}
$$

It is easy to see that $S$ is a "horned" 2 -sphere and hence is wildly imbedded in $E^{3}$. $A_{0}$ is contained in the union of $S$ and the bounded component of $E^{3}-S$, and for each component $C$ of $A_{0}$ the set $C \cap S$ consists of the endpoints of $C$.

If $p \in A_{0} \cap S$, then we can pierce $S$ at $p$ by extending the component $C$ of $A_{0}$ which contains $p$. On the other hand, if $p \in S-A_{0}$, then $S$ is locally polyhedral at $p$ and can certainly be pierced by a line segment at $p$.

In view of the above example, $\mathrm{R}$. H. Bing has raised the following two questions:

(1) Is a topological 2-sphere $S$ in $E^{3}$ tame if corresponding to each point $p \in S$ there are Euclidean (round) spheres $\sigma_{1}$ and $\sigma_{2}$ containing $p$ such that $\sigma_{1}-p$ and $\sigma_{2}-p$ lie on opposite sides of $S$ ?

Received by the editors September 8, 1962.

1 This work was supported by NSF Grant G23790. 
(2) Is a topological 2-sphere $S$ in $E^{3}$ tame if corresponding to each point $p \in S$ there are cones $\gamma_{1}$ and $\gamma_{2}$, each with vertex at $p$, such that $\gamma_{1}-p$ and $\gamma_{2}-p$ lie on opposite sides of $S$ ?

\section{BibliogRAPHY}

1. R. H. Bing, $A$ decomposition of $E^{8}$ into points and tame arcs such that the decomposition space is topologically different from $E^{3}$, Ann. of Math. (2) 65 (1957), 484-500.

2. M. K. Fort, Jr., A note concerning a decomposition space defined by Bing, Ann. of Math. (2) 65 (1957), 501-504.

UNIVERSITY OF GEORGIA

\section{CORRECTION TO "A CHARACTERIZATION OF QF-3 ALGEBRAS"}

\section{HIROYUKI TACHIKAWA}

J. P. Jans is kind enough to inform me a gap of Necessity proof in my paper appearing in these Proceedings, 13 (1962), 701-703. In this note I shall report Theorem 2 in the paper is however valid by a slight alteration of the proof. In p. 702, the argument between line 9 and line 18 should be replaced by the following: Let $e_{\lambda}$ be a primitive idempotent of $A$ such that $l(N) e_{\lambda} \neq 0$. Then there exists an element $x \in L$ such that $l(N) e_{\lambda} x \neq 0$ for $L$ is faithful. Denote $x$ by $\sum_{\kappa \neq \lambda} a_{\kappa} e_{\kappa}+a_{\lambda} e_{\lambda}, a_{\kappa}, a_{\lambda} \in A$. Since $e_{\lambda}\left(\sum_{\kappa \neq \lambda} a_{\kappa} e_{\kappa}\right) \subseteq N, l(N) e_{\lambda} x$ $=l(N) e_{\lambda} a_{\lambda} e_{\lambda}$ and we have $l(N) e_{\lambda} L e_{\lambda} \neq 0$. Here, suppose $L e_{\lambda} \neq A e_{\lambda}$. Then $L e_{\lambda} \subseteq N e_{\lambda}$ for $N e_{\lambda}$ is the unique maximal left ideal of $A e_{\lambda}$ and it follows $l(N) e_{\lambda} L e_{\lambda} \subseteq l(N) N=0$. But this is a contradiction. Thus we obtain $L e_{\lambda}=A e_{\lambda}$. Now, let $\theta$ be the epimorphism: $L \rightarrow L e_{\lambda}\left(=A e_{\lambda}\right)$, defined by $\theta(x)=x e_{\lambda}$ for all $x \in L$. Since $L e_{\lambda}$ is projective, we have a direct sum decomposition of $L: L_{\lambda} \oplus L_{\lambda}^{\prime}$, where $L_{\lambda} \approx A e_{\lambda}$. Then as $\operatorname{Hom}(L, K)$ is monomorphic to $P$ and $\operatorname{Hom}\left(A e_{\lambda}, K\right)$ is injective, $\operatorname{Hom}\left(A e_{\lambda}, K\right)$ is isomorphic to a direct summand of $P$. Thus if we denote by $\Lambda$ the set of all indices $\lambda$ such that $l(N) e_{\lambda} \neq 0$, $\operatorname{Hom}\left(\sum_{\lambda \in \Lambda} A e_{\lambda}, K\right)$ is projective. 